\title{
Pre-operative echocardiography among patients with coronary artery disease in the United States Veterans Affairs healthcare system: A retrospective cohort study
}

Emily B. Levitan ${ }^{1,2 *}$, Laura A. Graham ${ }^{1,2}$, Javier A. Valle ${ }^{3}$, Joshua S. Richman²,4, Robert Hollis ${ }^{2,4}$, Carla N. Holcomb ${ }^{2,4}$, Thomas M. Maddox ${ }^{3,5}$ and Mary T. Hawn 2,6

\begin{abstract}
Background: Echocardiography is not recommended for routine pre-surgical evaluation but may have value for patients at high risk of major adverse cardiovascular events (MACE). The objective of this study was to evaluate whether pre-operative echocardiography is associated with lower risk of post-operative MACE among patients with coronary artery disease.
\end{abstract}

Methods: Using administrative and registry data, we examined associations of echocardiography within 3 months prior to surgery with postoperative MACE (myocardial infarction, revascularization, or death within 30 days) among patients with coronary artery disease undergoing elective, non-cardiac surgeries in the United States Veterans Affairs healthcare system in 2000-2012.

Results: Echocardiography preceded 4,378 (16.4\%) of 26,641 surgeries. MACE occurred within 30 days following 944 (3.5 \%) surgeries. A $10 \%$ higher case-mix adjusted rate of pre-operative echocardiography assessed at the hospital level was associated with a hospital-level risk of MACE that was $1.0 \%$ (95\% confidence interval [CI] $0.1 \%, 2.0 \%$ ) higher overall and $1.7 \%$ (95 \% Cl 0.2 \%, 3.2\%) higher among patients with recent myocardial infarction, valvular heart disease, or heart failure. At the patient level, pre-operative echocardiography was associated with an odds ratio for MACE of $1.9(95 \% \mathrm{Cl} 1.7,2.2)$ overall and $1.8(95 \% \mathrm{Cl} 1.5,2.2)$ among patients with recent myocardial infarction, valvular heart disease, or heart failure adjusting for MACE risk factors.

Conclusions: Pre-operative echocardiography was not associated with lower risk of post-operative MACE, even in a high risk population. Future guidelines should encourage pre-operative echocardiography only in specific patients with cardiovascular disease among whom findings can be translated into effective changes in care.

Keywords: Echocardiography, Surgery, Major adverse cardiac events

Abbreviations: $\mathrm{Cl}$, Confidence interval; CPT, Current procedure terminology; CVD, Cardiovascular disease; HF, Heart failure; ICD9, International classification of diseases $9^{\text {th }}$ edition; MI, Myocardial infarction; VA, Veterans affairs; VASQIP, Veterans Affairs Surgical Quality Improvement Program; VHD, Valvular heart disease

\footnotetext{
* Correspondence: elevitan@uab.edu

${ }^{1}$ Department of Epidemiology, University of Alabama at Birmingham,

35294-0022 Birmingham, AL, USA

2Birmingham Veterans Affairs Medical Center, Birmingham, AL, USA

Full list of author information is available at the end of the article
} 


\section{Background}

Echocardiography is commonly performed prior to surgery [1], and abnormalities detected through echocardiography may be associated with higher risk of major adverse cardiac events (MACE) following surgery [2, 3]. Although the information obtained through echocardiography could inform care, in the general surgical population, pre-operative echocardiography provides little additional prognostic information, and its usefulness in guiding peri-operative treatment is unclear [2, 3]. For this reason, echocardiography is not recommended for routine pre-operative evaluation among patients without cardiovascular disease (CVD) [2-5]. In previous studies, pre-operative echocardiography has been associated with higher 30-day and 1-year mortality and longer length of stay $[1,6]$, which may be a result of higher risk patients being more likely to receive echocardiography. Additional testing, treatments, or treatment delays or cancellations triggered by echocardiography results may also contribute to these observations.

Preexisting CVDs, particularly heart failure (HF), valvular heart disease (VHD), and recent myocardial infarction (MI), are risk factors for MACE following non-cardiac surgery [3], and the proportion of surgical patients with CVD, including HF, VHD, and recent MI, is increasing [7]. Echocardiography is well-established as a valuable tool for diagnosis and evaluation of symptoms of many CVDs, and pre-operative echocardiography may be helpful for guiding peri-operative care among patients with established CVD $[2,3,5,7]$. We therefore hypothesized that pre-operative echocardiography is associated with a reduced risk of post-operative MACE among patients with coronary artery disease, particularly those who also have HF, VHD, or recent MI.

We examined the associations echocardiography performed in the 3 months prior to surgery (hereafter called pre-operative echocardiography) with post-operative MACE among patients with coronary artery disease undergoing elective, non-cardiac surgery in the United States Veterans Affairs (VA) healthcare system. Because concerning findings on echocardiography may cause patients and clinicians to avoid surgery, we additionally examined the correlations between echocardiography use, surgical volume, and surgery cancellations.

\section{Methods}

\section{Study population}

We conducted a retrospective cohort study within the VA healthcare system during fiscal years 2000-2012. The study protocol was reviewed and approved by the local VA Institutional Review Board of each co-author and conforms to the ethical guidelines of the 1975 Declaration of Helsinki. Because this study included only data routinely collected as part of clinical care, the Institutional Review Boards approved a waiver of informed consent.
The study population has been previously described [8]. This cohort was initially assembled to examine MACE following non-cardiac surgery in patients within 2 years of placement of a coronary stent [8]. Briefly, patients undergoing placement of coronary stents within the VA healthcare system during fiscal years 2000 through 2012 were identified using International Classification of Diseases $9^{\text {th }}$ edition (ICD9) procedure codes 36.06 for bare metal stents and 36.07 for drug eluting stents in VA administrative data. Non-cardiac surgery in the 2 years following stent placement was identified through the VA Surgical Quality Improvement Program (VASQIP) registry. For each patient with a stent, 2 patients without stents undergoing noncardiac surgery were included, matched on fiscal year of surgery, age within 5 years, work relative value unit (a measure of surgery complexity) within 5 units, surgical specialty, pre-operative creatinine $>2 \mathrm{mg} / \mathrm{dl}$, classification of high risk surgery (suprainguinal, intrathoracic, or intraperitoneal operations), and the components that make up the Revised Cardiac Risk Index (coronary artery disease, HF, stroke, chronic kidney disease, and insulindependent diabetes) [9]. All of the patients had a history of coronary artery disease. For the current study, we further limited the cohort to 8,823 stent/surgery patients and 17,818 matched surgery-only patients undergoing elective surgeries. Patients could be included in the cohort more than once if they had multiple surgeries. Patients with a history of MI in the 6 months prior to surgery or a history of HF or valvular disease in the past 24 months were considered a particularly high risk population. To examine the association between echocardiography and surgical volume and number of surgery cancellations, we examined a cohort of 126,773 individuals who had coronary stents placed, identified as described above.

\section{Pre-operative echocardiography}

Echocardiography within the 3 months prior to surgery was identified by Current Procedure Terminology (CPT) codes (93312, 93315, 93318, 93350, 93351, 93303, 93304, 93306, 93307, 93308, 93320, 93321, 93325) and ICD9 procedure codes (88.72) in administrative data. Because a recent echocardiogram may influence the decision to perform another echocardiogram, we also identified echocardiography in the 3 to 12 months prior to surgery.

\section{Covariates}

Covariates of interest were obtained from VASQIP (demographics, social factors, pre-operative comorbidities, and operative characteristics). Additional comorbidities were identified from the administrative data based on ICD9 diagnosis codes. For patients with surgeries in fiscal year 2005 and later, prescriptions for beta-blockers, statins, aspirin, clopidogrel, warfarin, renin-angiotensin system inhibitors, calcium channel blockers, diuretics, amiodarone, 
digoxin, and nitrates extending into or through the 30 days prior to surgery were identified using VA pharmacy data.

\section{Post-operative MACE}

MACE within 30 days following non-cardiac surgery was defined as an MI recorded in VASQIP or administrative data, revascularization recorded in administrative data, or death recorded in VASQIP or administrative data. This definition of MACE has been used previously in this population [8].

\section{Statistical analysis}

Patient and operative characteristics were compared by risk group and receipt of pre-operative echocardiography. Tests for differences conducted using Chi-square tests for proportions and Wilcoxon Rank Sum for continuous variables. These analyses were performed using SAS version 9.3.

For hospitals with at least 25 included surgeries, hospitallevel echocardiography rates were calculated as the percentage of patients who received an echocardiogram in the 3 months prior to surgery. Of the 146 VA hospitals, we included 117 with at least 25 surgeries in the overall population, 87 with at least 25 surgeries in the population with recent MI, HF, or VHD, and 117 with at least 25 surgeries in the population without recent MI, HF, or VHD. To adjust the hospital-level echocardiography rates for case-mix, we constructed mixed-effects logistic regression models with the outcome of pre-operative echocardiography, random intercepts for the hospitals, and patient and operative characteristics as the independent variables [10]. Values of the random intercepts were calculated using empirical Bayesian estimates [11], and case-mix adjusted echocardiography rates for each hospital were calculated using the intercept for that hospital and the mean values of the patient and operative characteristics across hospitals. Observed and case-mix adjusted hospital-level echocardiography rates were plotted using a density plot. These analyses were repeated stratified by MACE risk group. Plots were developed using the R-program statistical software and GGPLOT2 package [12].

Linear regression was used to model MACE risk differences associated with a $10 \%$ higher hospital-level rate of pre-operative echocardiography and by quartiles of hospital-level rate of pre-operative echocardiography. Models were repeated for case-mix adjusted rate of pre-operative echocardiography. The association between patient-level receipt of pre-operative echocardiography and MACE following surgery was estimated using logistic regression with a random intercept for hospital. Backwards stepwise selection was used to identify covariates from the patient and operative characteristics expected to be associated with echocardiography and/or MACE. All models were constructed using R-program statistical software and the lme4 package [13, 14].
In the cohort of patients who received a coronary stent, we calculated the hospital-level correlation between rate of pre-operative echocardiography use and surgical volume and number of surgery cancellations in the 2 years following coronary stent placement. Additionally, we examined the percentage of planned surgeries which were cancelled in individuals with echocardiography in the 3 months prior to the planned surgery and the percentage in the overall population.

\section{Results}

Among 26,641 elective, non-cardiac surgeries, 4,378 (16.4\%) had pre-operative echocardiography (Table 1). Among patients with high-risk conditions (recent MI, HF, or VHD), $28.7 \%$ had pre-operative echocardiography, compared to $12.8 \%$ of patients without those conditions. The median time between echocardiography and surgery was 35 days (interquartile range 10-63 days). Patients who received pre-operative echocardiography were more likely to be $\geq 60$ years of age and to have a history of diabetes, peripheral vascular disease, coronary artery bypass graft, chronic kidney disease, arrhythmia, and pacemakers. Patients with coronary stents were slightly less likely to have pre-operative echocardiography than patients without stents. Compared to patients without pre-operative echocardiography, the patients with pre-operative echocardiography were more likely to have inpatient surgery, higher work relative value units, high risk surgeries, and, among the group with coronary stents, a shorter time from stenting to surgery.

Before adjusting for case-mix, VA hospitals varied widely in the use of pre-operative echocardiography (mean $13.8 \%$, range $0.0 \%-30.6 \%$, standard deviation $6.8 \%$ ) (Fig. 1). After adjusting for case-mix, the variability decreased (mean $14.3 \%$, range 8.6-25.5 \%, standard deviation $3.1 \%$ ). Hospitals were more variable in the use of pre-operative echocardiography among patients with recent MI, HF, or VHD than among patients without these conditions. There was a moderate correlation $(r=0.53)$ between hospital-level use of pre-operative echocardiography among patients with and without recent MI, HF, or VHD (Additional file 1: Figure S1).

In the 30 days following non-cardiac surgery, 944 patients $(3.5 \%)$ experienced MACE $(7.5 \%$ with preoperative echocardiography versus $2.8 \%$ without preoperative echocardiography, $\mathrm{p}<0.001$ ). Before adjusting for case-mix, higher hospital rates of pre-operative echocardiography were associated with higher hospital rates of MACE within 30 days of surgery (Fig. 2). For every $10 \%$ higher rate of pre-operative echocardiography, the hospital-level 30-day risk of MACE was $1.25 \%$ (95\% confidence interval $[\mathrm{CI}] 0.77 \%, 1.74 \%$ ) higher. Associations between echocardiography and MACE were stronger comparing patients with $\mathrm{HF}, \mathrm{VHD}$, or recent MI to patients 
Table 1 Characteristics of surgical patients in the Veterans Affairs health system by whether or not they receive pre-operative echocardiography ${ }^{a}$

\begin{tabular}{|c|c|c|c|c|c|c|c|c|c|}
\hline \multirow[b]{2}{*}{ Echocardiogram in the 3 months prior to surgery } & \multicolumn{4}{|c|}{$\begin{array}{l}\text { Patients with heart failure, valvular heart } \\
\text { disease, recent myocardial infarction }\end{array}$} & \multicolumn{4}{|c|}{$\begin{array}{l}\text { Patients without heart failure, valvular heart } \\
\text { disease, recent myocardial infarction }\end{array}$} & \multirow[b]{2}{*}{$P$-Value } \\
\hline & \multicolumn{2}{|c|}{ Yes $(N=1,732)$} & \multicolumn{2}{|c|}{ No $(N=4,307)$} & \multicolumn{2}{|c|}{ Yes $(N=2,646)$} & \multicolumn{2}{|c|}{ No $(N=17,956)$} & \\
\hline \multicolumn{10}{|l|}{ Demographics } \\
\hline \multicolumn{10}{|l|}{ Age } \\
\hline Years, Median (25th percentile-75th percentile) & 67.0 & $(61.0-75.0)$ & 67.0 & $(60.0-75.0)$ & 65.0 & $(60.0-73.0)$ & 64.0 & $(59.0-72.0)$ & $<0.001$ \\
\hline$<60$ & 339 & (19.6) & 968 & $(22.5)$ & 601 & $(22.7)$ & 4,949 & $(27.6)$ & $<0.001$ \\
\hline$\geq 60$ & 1,393 & $(80.4)$ & 3,339 & $(77.5)$ & 2,045 & $(77.3)$ & 13,007 & (72.4) & \\
\hline \multicolumn{10}{|l|}{ Race } \\
\hline Black & 194 & $(11.5)$ & 451 & $(10.8)$ & 315 & $(12.3)$ & 1,910 & $(11.0)$ & 0.11 \\
\hline Other & 25 & $(1.5)$ & 54 & $(1.3)$ & 32 & $(1.3)$ & 177 & $(1.0)$ & \\
\hline White & 1,471 & $(87.0)$ & 3,686 & $(88.0)$ & 2,207 & (86.4) & 15,321 & (88.0) & \\
\hline \multicolumn{10}{|l|}{ Gender } \\
\hline Female & 27 & $(1.6)$ & 94 & $(2.2)$ & 40 & $(1.5)$ & 401 & $(2.2)$ & 0.04 \\
\hline Male & 1,705 & (98.4) & 4,213 & (97.8) & 2,606 & $(98.5)$ & 17,555 & (97.8) & \\
\hline \multicolumn{10}{|l|}{ Health status } \\
\hline \multicolumn{10}{|l|}{ Echocardiogram in the 3 to 12 Months Prior to Surgery } \\
\hline No & 1,105 & $(63.8)$ & 2,318 & $(53.8)$ & 2,254 & $(85.2)$ & 14,561 & $(81.1)$ & $<0.001$ \\
\hline Yes & 627 & $(36.2)$ & 1,989 & $(46.2)$ & 392 & $(14.8)$ & 3,395 & $(18.9)$ & \\
\hline \multicolumn{10}{|l|}{ Myocardial infarction within 6 months } \\
\hline No & 997 & $(57.6)$ & 2,465 & $(57.2)$ & 2,646 & $(100.0)$ & 17,956 & $(100.0)$ & $<0.001$ \\
\hline Yes & 735 & $(42.4)$ & 1,842 & $(42.8)$ & 0 & $(0.0)$ & 0 & $(0.0)$ & \\
\hline \multicolumn{10}{|l|}{ Heart failure hospitalization within 6 months } \\
\hline No & 1,108 & $(64.0)$ & 3,115 & $(72.3)$ & 2,646 & $(100.0)$ & 17,956 & $(100.0)$ & $<0.001$ \\
\hline Yes & 624 & $(36.0)$ & 1,192 & $(27.7)$ & 0 & $(0.0)$ & 0 & $(0.0)$ & \\
\hline \multicolumn{10}{|l|}{ History of heart failure } \\
\hline No & 784 & $(45.3)$ & 2,381 & $(55.3)$ & 1,974 & $(74.6)$ & 15,444 & $(86.2)$ & $<0.001$ \\
\hline Yes & 948 & $(54.7)$ & 1,924 & $(44.7)$ & 672 & $(25.4)$ & 2,472 & (13.8) & \\
\hline \multicolumn{10}{|l|}{ Valvular heart disease } \\
\hline No & 882 & $(50.9)$ & 2,329 & $(54.1)$ & 2,646 & $(100.0)$ & 17,956 & $(100.0)$ & $<0.001$ \\
\hline Yes & 850 & $(49.1)$ & 1,978 & $(45.9)$ & 0 & $(0.0)$ & 0 & $(0.0)$ & \\
\hline \multicolumn{10}{|l|}{ Coronary artery stents } \\
\hline None & 918 & $(53.0)$ & 2,231 & $(51.8)$ & 1,905 & $(72.0)$ & 12,764 & $(71.1)$ & $<0.001$ \\
\hline Bare metal stents & 433 & $(25.0)$ & 1,222 & $(28.4)$ & 382 & $(14.4)$ & 2,563 & $(14.3)$ & \\
\hline Drug-eluting stents & 371 & $(21.4)$ & 830 & $(19.3)$ & 350 & $(13.2)$ & 2,563 & $(14.3)$ & \\
\hline Both bare metal and drug-eluting stents & 10 & $(0.6)$ & 24 & $(0.6)$ & 9 & (0.3) & 66 & $(0.4)$ & \\
\hline \multicolumn{10}{|l|}{ Diabetes } \\
\hline None & 1,031 & $(59.5)$ & 2,830 & $(65.7)$ & 1,723 & $(65.1)$ & 12,360 & $(68.8)$ & $<0.001$ \\
\hline Non-insulin dependent & 308 & $(17.8)$ & 754 & $(17.5)$ & 483 & $(18.3)$ & 3,165 & $(17.6)$ & \\
\hline Insulin dependent & 393 & $(22.7)$ & 723 & $(16.8)$ & 440 & $(16.6)$ & 2,431 & $(13.5)$ & \\
\hline \multicolumn{10}{|l|}{ Peripheral vascular disease } \\
\hline No & 1,063 & (61.4) & 2,854 & $(66.3)$ & 1,760 & $(66.5)$ & 13,772 & (76.7) & $<0.001$ \\
\hline Yes & 669 & (38.6) & 1,453 & $(33.7)$ & 886 & $(33.5)$ & 4,184 & $(23.3)$ & \\
\hline
\end{tabular}


Table 1 Characteristics of surgical patients in the Veterans Affairs health system by whether or not they receive pre-operative echocardiography ${ }^{\text {a }}$ (Continued)

\begin{tabular}{|c|c|c|c|c|c|c|c|c|c|}
\hline \multicolumn{10}{|l|}{ Stroke } \\
\hline No & 1,681 & $(97.1)$ & 4,230 & $(98.3)$ & 2,544 & $(96.2)$ & 17,673 & $(98.6)$ & $<0.001$ \\
\hline Yes & 51 & $(2.9)$ & 75 & $(1.7)$ & 102 & (3.9) & 243 & (1.4) & \\
\hline \multicolumn{10}{|l|}{ Hypertension } \\
\hline No & 117 & $(9.2)$ & 277 & $(10.1)$ & 196 & $(9.9)$ & 1,772 & $(14.2)$ & $<0.001$ \\
\hline Yes & 1,154 & $(90.8)$ & 2,458 & (89.9) & 1,784 & $(90.1)$ & 10,749 & $(85.9)$ & \\
\hline \multicolumn{10}{|l|}{ Coronary artery bypass grafting } \\
\hline No & 1,600 & $(92.4)$ & 4,024 & $(93.4)$ & 2,554 & $(96.5)$ & 17,467 & $(97.3)$ & $<0.001$ \\
\hline Yes & 132 & (7.6) & 283 & (6.6) & 92 & (3.5) & 489 & (2.7) & \\
\hline \multicolumn{10}{|l|}{ Chronic kidney disease } \\
\hline No & 1,268 & $(73.2)$ & 3,514 & (81.6) & 2,229 & $(84.2)$ & 16,083 & (89.6) & $<0.001$ \\
\hline Yes & 464 & $(26.8)$ & 793 & $(18.4)$ & 417 & $(15.8)$ & 1,873 & $(10.4)$ & \\
\hline \multicolumn{10}{|l|}{ Arrhythmia } \\
\hline No & 789 & $(45.6)$ & 2,207 & $(51.2)$ & 1,681 & $(63.5)$ & 12,899 & $(71.8)$ & $<0.001$ \\
\hline Yes & 943 & $(54.5)$ & 2,100 & $(48.8)$ & 965 & $(36.5)$ & 5,057 & $(28.2)$ & \\
\hline \multicolumn{10}{|l|}{ Pacemaker } \\
\hline No & 1,686 & $(97.3)$ & 4,225 & $(98.1)$ & 2,618 & $(98.9)$ & 17,828 & $(99.3)$ & $<0.001$ \\
\hline Yes & 46 & $(2.7)$ & 82 & (1.9) & 28 & (1.1) & 128 & $(0.7)$ & \\
\hline \multicolumn{10}{|l|}{ Cardioverter defibrillator } \\
\hline No & 1,697 & $(98.0)$ & 4,235 & (98.3) & 2,629 & $(99.4)$ & 17,870 & $(99.5)$ & $<0.001$ \\
\hline Yes & 35 & $(2.0)$ & 72 & (1.7) & 17 & (0.6) & 86 & $(0.5)$ & \\
\hline \multicolumn{10}{|l|}{ Operative Characteristics } \\
\hline \multicolumn{10}{|l|}{ Inpatient/Outpatient, n (\%) } \\
\hline Outpatient & 456 & $(26.4)$ & 1,536 & $(35.8)$ & 751 & $(28.5)$ & 8,092 & $(45.1)$ & $<0.001$ \\
\hline Inpatient & 1,273 & $(73.6)$ & 2,761 & $(64.3)$ & 1,886 & $(71.5)$ & 9,837 & $(54.9)$ & \\
\hline \multicolumn{10}{|l|}{ Work Relative Value Unit } \\
\hline Median (25th percentile-75th percentile) & 18.2 & $(11.8-22.6)$ & 15.4 & $(9.6-21.8)$ & 19.6 & $(11.8-23.3)$ & 15.3 & $(8.4-21.8)$ & $<0.001$ \\
\hline$<10$ & 762 & $(44.0)$ & 1,887 & $(43.8)$ & 1,124 & $(42.5)$ & 6,821 & $(38.0)$ & $<0.001$ \\
\hline $10-20$ & 372 & $(21.5)$ & 1,177 & $(27.3)$ & 514 & $(19.4)$ & 5,847 & $(32.6)$ & \\
\hline$>20$ & 597 & $(34.5)$ & 1,243 & (28.9) & 1,008 & $(38.1)$ & 5,288 & $(29.5)$ & \\
\hline \multicolumn{10}{|l|}{ High Risk Surgery } \\
\hline No & 1,286 & $(74.3)$ & 3,332 & $(77.4)$ & 1,909 & $(72.2)$ & 14,417 & $(80.3)$ & $<0.001$ \\
\hline Yes & 446 & $(25.8)$ & 975 & (22.6) & 737 & (27.9) & 3,539 & $(19.7)$ & \\
\hline \multicolumn{10}{|l|}{ Procedure Type } \\
\hline Digestive & 402 & $(23.2)$ & 1,141 & (26.5) & 593 & (22.4) & 4,959 & $(27.6)$ & $<0.001$ \\
\hline Eye/Ear/Skin & 31 & (1.8) & 85 & (2.0) & 38 & (1.4) & 299 & (1.7) & \\
\hline Genital/Urinary & 206 & (11.9) & 607 & $(14.1)$ & 285 & $(10.8)$ & 2,616 & $(14.6)$ & \\
\hline Musculoskeletal & 272 & $(15.7)$ & 828 & $(19.2)$ & 475 & $(18.0)$ & 4,465 & (24.9) & \\
\hline Nervous & 34 & $(2.0)$ & 119 & (2.8) & 88 & (3.3) & 709 & (4.0) & \\
\hline Other & 50 & $(2.9)$ & 102 & (2.4) & 78 & (3.0) & 422 & $(2.4)$ & \\
\hline Respiratory & 82 & $(4.7)$ & 146 & (3.4) & 156 & $(5.9)$ & 509 & $(2.8)$ & \\
\hline Vascular & 655 & $(37.8)$ & 1,279 & (29.7) & 933 & (35.3) & 3,977 & $(22.2)$ & \\
\hline
\end{tabular}


Table 1 Characteristics of surgical patients in the Veterans Affairs health system by whether or not they receive pre-operative echocardiography ${ }^{a}$ (Continued)

\begin{tabular}{|c|c|c|c|c|c|c|c|c|c|}
\hline \multicolumn{10}{|l|}{ Time to Surgery Since Stent } \\
\hline Days, Median (25th percentile-75th percentile) & 132.0 & $(63.0-370.0)$ & 184.0 & $(104.5-433.0)$ & 347.0 & $(92.0-515.0)$ & 406.0 & $(251.0-552.0)$ & \\
\hline None & 100 & (5.8) & 130 & (3.0) & 79 & (3.0) & 173 & (1.0) & $<0.001$ \\
\hline$<6$ Weeks & 214 & $(12.4)$ & 287 & (6.7) & 103 & (3.9) & 266 & (1.5) & $<0.001$ \\
\hline 6 Weeks to 3 Months & 166 & (9.6) & 611 & $(14.2)$ & 41 & (1.6) & 341 & (1.9) & \\
\hline 3 to 6 Months & 123 & (7.1) & 389 & (9.0) & 169 & (6.4) & 1,405 & $(7.8)$ & \\
\hline 6 to 12 Months & 211 & (12.2) & 659 & (15.3) & 349 & $(13.2)$ & 3,007 & (16.8) & \\
\hline$>12$ Months & 918 & (53.0) & 2,231 & (51.8) & 1,905 & (72.0) & 12,764 & (71.1) & \\
\hline
\end{tabular}

${ }^{a}$ Numbers in the table are median $\left(25^{\text {th }}\right.$ percentile- $75^{\text {th }}$ percentile) or $\mathrm{N}$ (column \%)

without these conditions. After adjusting for case-mix, associations were attenuated and without a clear pattern of risk across quartiles. For every $10 \%$ higher rate of preoperative echocardiography, the hospital-level 30-day risk of MACE was $1.02 \%$ (95\% CI $0.07 \%, 1.97 \%$ ) higher in the overall population and $1.73 \%$ (95\% CI $0.22 \%, 3.24 \%)$ higher in the high risk population.

In patient-level analyses, pre-operative echocardiography was associated with a higher risk of MACE at 30 days (adjusted odds ratio 1.92, $95 \% \mathrm{CI} 1.66,2.23)$ in the overall population after adjusting for risk factors for MACE (Fig. 3).

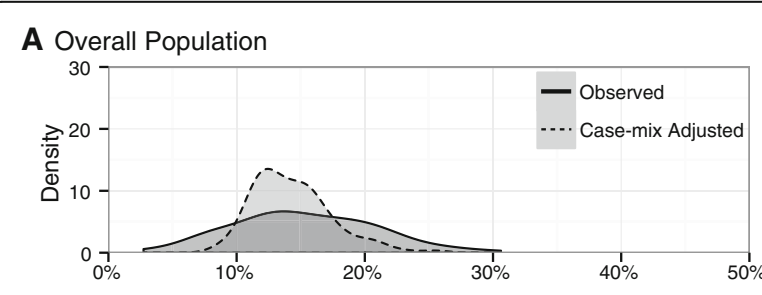

B High Risk Population

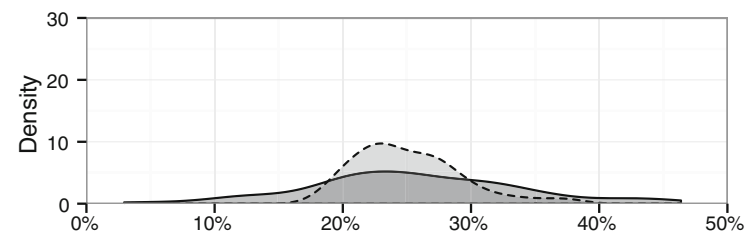

C Low Risk Population

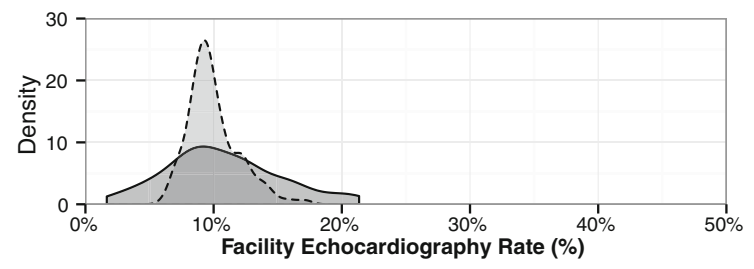

Fig. 1 Facility variation in use of pre-operative echocardiography in the Veterans Affairs Healthcare System. Smoothed distribution of pre-operative echocardiography rates by hospital. Of the 146 Veterans Affairs hospitals, 117 with at least 25 surgeries in the overall population, 87 with at least 25 surgeries in the population with recent Ml, HF, or $\mathrm{VHD}$, and 117 with at least 25 surgeries in the population without recent $\mathrm{Ml}, \mathrm{HF}$, or VHD were included
The higher risk of MACE associated with pre-operative echocardiography was observed in those with and without recent MI, HF, or VHD. In sensitivity analyses, results were consistent after adjusting for pre-operative medication use among patients who had surgeries in 2005-2012 (not shown).

Hospital-level use of echocardiography was not associated with hospital surgical volume $(r=-0.05, p=0.59)$ or hospital number of surgery cancellations $(r=-0.03, p=$ 0.74 ) in patients with coronary stents. The proportion of surgeries cancelled was $25 \%$ in patients who received pre-operative echocardiography compared to an average cancellation rate of $21 \%$. The majority of cancellations (90.6\%) occurred within 7 days of the planned surgery date with $51.7 \%$ occurring on the same day and $80.6 \%$ occurring within $48 \mathrm{~h}$.

\section{Discussion}

In this population of veterans with coronary artery disease, $16 \%$ underwent echocardiography in the 3 months prior to elective, non-cardiac surgery $(29 \%$ among patients with recent MI, VHD, or HF). Contrary to our hypothesis, preoperative echocardiography was not associated with lower risk of post-operative MACE either in the overall population or in those with recent MI, HF, or VHD. In fact, both hospital-level and patient-level use of pre-operative echocardiography was associated with a higher risk of MACE.

Our results are consistent with 2 previous studies in general surgical populations. In the United States Medicare population, patients who received tests of ventricular function, including echocardiography, had higher 30-day and 1year mortality following vascular surgery than patients who did not [6]. In a population-based study from Ontario, preoperative echocardiography was associated with increased risk of 30-day and 1-year mortality and longer length of stay, even among patients with HF [1]. It is possible that patients would have had even higher risk of MACE without changes to management initiated following echocardiography or that the higher risk associated with echocardiography reflects patient risk factors that were not fully 


\section{0-Day MACE Risk \\ Crude Case-mix adjusted}

\section{Overall population}

Quartile 1 (Reference)

Quartile 2

Quartile 3

Quartile 4

Patients with HF, VHD, or recent M

Quartile 1 (Reference)

Quartile 2

Quartile 3

Quartile 4

Patients without HF, VHD, or recent MI

Quartile 1 (Reference)

Quartile 2

Quartile 3

Quartile 4

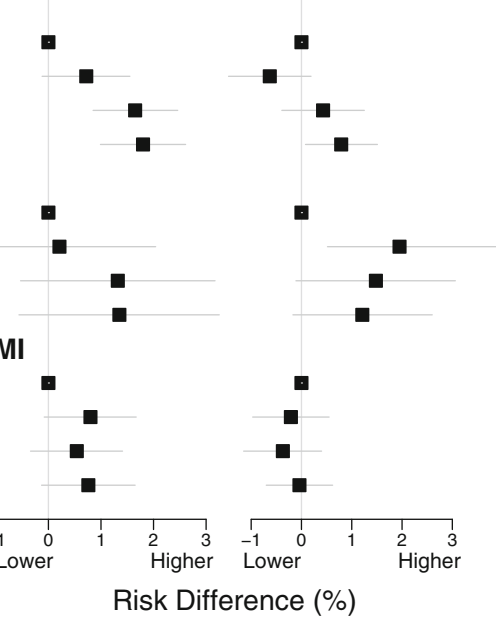

Fig. 2 Association between hospital-level variation in pre-operative echocardiography and post-operative major adverse cardiac events. MACE: Major adverse cardiac event, HF: Heart failure, VHD: Valvular heart disease, Ml: Myocardial infarction. Quartile ranges: Overall population unadjusted $\leq 9.72 \%$, 10.00-14.06 \%, 14.19-18.84 \%, and $\geq 19.01 \%$; Overall population case mix-adjusted $\leq 12.70 \%, 12.75-14.20 \%, 14.25-15.97 \%$, and $\geq 16.00 \%$; Patients with heart failure, valvular heart disease, recent myocardial infarction unadjusted $\leq 18.18 \%, 18.46-24.86 \%, 25.00-30.43 \%$, and $\geq 31.11 \%$; Patients with heart failure, valvular heart disease, recent myocardial infarction case mix-adjusted $\leq 23.45 \%, 23.47-25.11 \%, 25.11-27.23 \%$, and $\geq 27.49 \%$; Patients without heart failure, valvular heart disease, recent myocardial infarction unadjusted $\leq 6.06 \%, 6.11-9.85 \%, 9.88-12.94 \%$, and $\geq 12.95 \%$; Patients without heart failure, valvular heart disease, recent myocardial infarction case mix-adjusted $\leq 9.04 \%$, 9.04-9.67 \%, 9.67-10.52\%, and $\geq 10.53 \%$

accounted for in statistical analysis. However, it is also possible that echocardiography led to changes or delays in care that could have contributed to the increased risk of MACE. In a prior study, patients who received preoperative echocardiography were more likely to receive new prescriptions for beta-blockers, statins, and reninangiotensin system inhibitors [1]. Although these medications can be beneficial for chronic management and may globally reduce cardiovascular risk, recent initiation may be harmful in some patients undergoing surgery [15].

Echocardiography is widely indicated for patients with suspected new or worsening CVD [2]. However, clinical guidelines have recommended against routine preoperative echocardiography in patients without CVD since at least 2002 [16]. In recent years, avoiding routine pre-operative echocardiography among patients without CVD has received particular attention as part of appropriate use criteria and the Choosing Wisely campaign because of increased costs and lack of value in guiding surgical care $[2-4,17]$. The guidelines and recommendations are less clear on the value of pre-operative echocardiography in patients with CVD, like the patients with coronary artery disease included in the current study. Additionally, guidelines do not always have a strong impact on patient care. A recent study of United States nationally representative data from 1997-2010 found that there was little change in the routine utilization of pre-operative radiography, hematocrit, urinalysis, and cardiac stress testing following

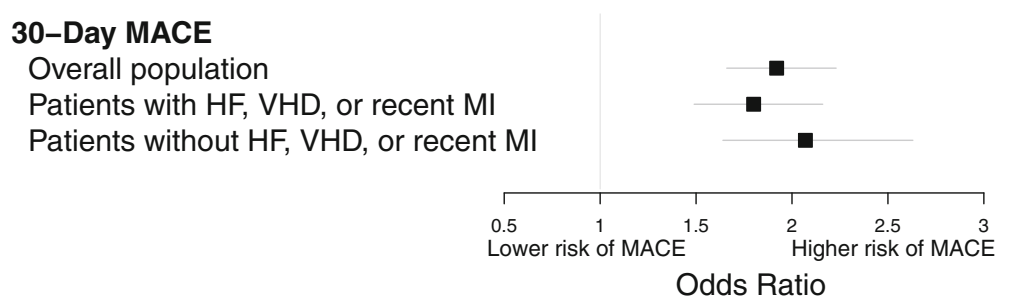

Fig. 3 Association between patient level pre-operative echocardiography and post-operative major adverse cardiac events. MACE: Major adverse cardiac event, HF: Heart failure, VHD: Valvular heart disease, Ml: Myocardial infarction. Models included a random intercept for hospital and adjusted for echocardiogram during the 3 to 12 months prior to surgery, age at surgery, recent myocardial infarction, history of heart failure, history of chronic kidney disease, history of arrhythmia, surgery case status, surgery admission status, work relative value unit, year of surgery, procedure type, and an indicator for whether the surgery was a high risk procedure 
recommendations against their use, but pre-operative electrocardiogram use did decline after professional societies recommended against it [18].

This study had several strengths, include the large population treated in an integrated health system. However, there are also important limitations to this observational, non-randomized study that prevent us from drawing conclusions about the causal effect of pre-operative echocardiography. Although we adjusted for MACE risk factors through case-mix adjustment in hospital-level analysis and multivariable models in patient-level models, there may be residual confounding by indication, where patients who receive the test have higher underlying risk. We did not find associations between use of pre-operative echocardiography and cancellations of surgeries. Nor did we find an association between hospital pre-operative echocardiography use and surgical volume. However, we could only detect cancellations of schedule surgeries; echocardiography could have resulted in surgeries never being scheduled. We could not determine whether echocardiography was performed as part of a pre-operative evaluation or for another clinical indication. In addition, results of the echocardiograms were not available. Because the data came from the VA health system, there were few women included.

\section{Conclusions}

In conclusion, pre-operative echocardiography was common among this population of patients with coronary artery disease undergoing elective, non-cardiac surgery. Pre-operative echocardiography was not associated with a lower risk of post-operative MACE either in the overall population or in the high risk subgroup with recent MI, VHD, or HF. These results suggest that routine pre-operative echocardiography may not reduce MACE even in high risk populations. Future guidelines should encourage pre-operative echocardiography only in specific patients among whom findings can be translated into effective changes in care.

\section{Additional file}

Additional file 1: Figure S1. Hospital-level variation in preoperative echocardiography by risk of post-operative major adverse cardiac events. (PDF $439 \mathrm{~kb}$ )

\section{Acknowledgements}

Not applicable.

\section{Funding}

This study was supported by a United States VA Health Services Research \& Development grant (No. IIR 09-347). In addition, Drs Maddox and Richman are supported by VA Career Development Awards. The funding agency did not have a role in the design of the study and collection, analysis, and interpretation of data or in writing the manuscript.

\section{Availability of data and material}

The data that support the findings of this study are available from the United States Veterans Affairs healthcare system but restrictions apply to the availability of these data, and so are not publicly available. Data are however available from the authors upon reasonable request and with permission of United States Veterans Affairs healthcare system.

\section{Authors' contributions}

EBL designed the statistical analysis, lead the interpretation of research findings, and wrote the manuscript. LAG collected data, build datasets, conducted statistical analysis, and revised the manuscript. JAV provided clinical cardiology expertise, advised on statistical analysis, and revised the manuscript. JSR provided input on the design of the statistical analysis, helped interpret results, and revised the manuscript. $\mathrm{RH}$ provided surgical expertise, provided feedback on study design, and revised the manuscript. $\mathrm{CNH}$ provided expertise on surgical practice in the Veterans Affairs system and revised the manuscript. TMM provided study oversight, provided expertise on cardiology practice in the Veterans Affairs system, and revised the manuscript. MTH provided study oversight, designed the parent study from which this data was obtained, obtained funding for the study, provided surgical expertise, and revised the manuscript. All authors read and approved the final manuscript.

\section{Competing interests}

EBL reports research support from Amgen and has served as a consultant for Amgen and for Robinson Calcagnie Robinson Shapiro Davis unrelated to the topic of this work. The other authors report no competing interests.

\section{Consent for publication}

Not applicable.

\section{Ethics approval and consent to participate}

The study protocol was reviewed and approved by the local VA Institutional Review Board of each co-author and conforms to the ethical guidelines of the 1975 Declaration of Helsinki. Because this study included only data routinely collected as part of clinical care, the Institutional Review Boards approved a waiver of informed consent

\section{Author details}

'Department of Epidemiology, University of Alabama at Birmingham, 35294-0022 Birmingham, AL, USA. ²Birmingham Veterans Affairs Medical Center, Birmingham, AL, USA. ${ }^{3}$ Division of Cardiology, University of Colorado, Denver, CO, USA. ${ }^{4}$ Department of Surgery, University of Alabama at Birmingham, Birmingham, AL, USA. ${ }^{5}$ Veterans Affairs Eastern Colorado Health Care System, Denver, CO, USA. ${ }^{6}$ Department of Surgery, Stanford University, Stanford, CA, USA.

Received: 20 July 2016 Accepted: 26 August 2016

Published online: 05 September 2016

\section{References}

1. Wijeysundera DN, Beattie WS, Karkouti K, Neuman MD, Austin PC, Laupacis A. Association of echocardiography before major elective non-cardiac surgery with postoperative survival and length of hospital stay: population based cohort study. BMJ. 2011;342:d3695.

2. American College of Cardiology Foundation Appropriate Use Criteria Task F, American Society of E, American Heart A, American Society of Nuclear C, Heart Failure Society of A, Heart Rhythm S, Society for Cardiovascular A, Interventions, Society of Critical Care M, Society of Cardiovascular Computed T, et al. ACCF/ASE/ AHA/ASNC/HFSA/HRS/SCAI/SCCM/SCCT/SCMR 2011 Appropriate Use Criteria for Echocardiography. A Report of the American College of Cardiology Foundation Appropriate Use Criteria Task Force, American Society of Echocardiography, American Heart Association, American Society of Nuclear Cardiology, Heart Failure Society of America, Heart Rhythm Society, Society for Cardiovascular Angiography and Interventions, Society of Critical Care Medicine, Society of Cardiovascular Computed Tomography, and Society for Cardiovascular Magnetic Resonance Endorsed by the American College of Chest Physicians. J Am Coll Cardiol. 2011;57(9):1126-66.

3. Fleisher LA, Fleischmann KE, Auerbach AD, Barnason SA, Beckman JA, Bozkurt B, Davila-Roman VG, Gerhard-Herman MD, Holly TA, Kane GC, et al. ACC/AHA Guideline on Perioperative Cardiovascular Evaluation and Management of Patients Undergoing Noncardiac Surgery: A Report of the American College of Cardiology/American Heart Association Task Force on Practice Guidelines. J Am Coll Cardiol. 2014;64:e77-137. 
4. American Society of Echocardiography Five Things Physicians and Patients Should Question. 2013. http://www.choosingwisely.org/doctor-patient-lists/ american-society-of-echocardiography/. Accessed 24 Mar 2015.

5. Task Force for Preoperative Cardiac Risk A, Perioperative Cardiac Management in Non-cardiac S, European Society of C, Poldermans D, Bax JJ, Boersma E, De Hert S, Eeckhout E, Fowkes G, Gorenek B, et al. Guidelines for pre-operative cardiac risk assessment and perioperative cardiac management in non-cardiac surgery. Eur Heart J. 2009;30(22):2769-812.

6. Fleisher LA, Eagle KA, Shaffer T, Anderson GF. Perioperative- and long-term mortality rates after major vascular surgery: the relationship to preoperative testing in the medicare population. Anesth Analg. 1999;89(4):849-55.

7. Beattie WS, Wijeysundera DN. The growing burden of perioperative heart failure. Anesth Analg. 2014;119(3):506-8.

8. Hawn MT, Graham LA, Richman JS, Itani KM, Henderson WG, Maddox TM. Risk of major adverse cardiac events following noncardiac surgery in patients with coronary stents. JAMA. 2013;310(14):1462-72.

9. Lee TH, Marcantonio ER, Mangione CM, Thomas EJ, Polanczyk CA, Cook EF, Sugarbaker DJ, Donaldson MC, Poss R, Ho KKL, et al. Derivation and Prospective Validation of a Simple Index for Prediction of Cardiac Risk of Major Noncardiac Surgery. Circulation. 1999;100(10):1043-9.

10. Normand S-LT, Shahian DM: Statistical and Clinical Aspects of Hospital Outcomes Profiling. 2007:206-226.

11. Fitzmaurice GM, Laird NM, Ware JH. Applied Longitudinal Analysis. Hoboken, New Jersey: Wiley; 2004.

12. Wickham H. GGPLOT2: Elegant Graphics for Data Anlaysis. New York: Springer; 2009.

13. Bates $D$, Machler M, Bolker BM, Walker SC. Fitting linear mixed-effects models using Ime4. 2015. http://cran.r-project.org/web/packages/Ime4/ vignettes/Imer.pdf. Accessed 30 Jan 2015.

14. Bates D, Maechler M, Bolker B, Walker S. Ime4: Linear mixed-effects models using Eigen and S4. 2014. http://cran.r-project.org/web/packages//me4/ index.html. Accessed 30 Jan 2015.

15. Devereaux PJ, Yang H, Yusuf S, Guyatt G, Leslie K, Villar JC, Xavier D, Chrolavicius S, Greenspan L, Pogue J, et al. Effects of extended-release metoprolol succinate in patients undergoing non-cardiac surgery (POISE trial): a randomised controlled trial. Lancet. 2008;371(9627):1839-47.

16. Eagle KA, Berger PB, Calkins H, Chaitman BR, Ewy GA, Fleischmann KE, Fleisher LA, Froehlich JB, Gusberg RJ, Leppo JA, et al. ACC/AHA guideline update for perioperative cardiovascular evaluation for noncardiac surgeryexecutive summary a report of the American College of Cardiology/American Heart Association Task Force on Practice Guidelines (Committee to Update the 1996 Guidelines on Perioperative Cardiovascular Evaluation for Noncardiac Surgery). Circulation. 2002;105(10):1257-67.

17. Group TTACW, Douglas PS, Khandheria B, Stainback RF, Weissman NJ, Panel TTACT, Brindis RG, Patel MR, Alpert JS, Fitzgerald D, et al. ACCF/ASE/ACEP/ ASNC/SCAI/SCCT/SCMR 2007 appropriateness criteria for transthoracic and transesophageal echocardiography: a report of the American College of Cardiology Foundation Quality Strategic Directions Committee Appropriateness Criteria Working Group, American Society of Echocardiography, American College of Emergency Physicians, American Society of Nuclear Cardiology, Society for Cardiovascular Angiography and Interventions, Society of Cardiovascular Computed Tomography, and the Society for Cardiovascular Magnetic Resonance. Endorsed by the American College of Chest Physicians and the Society of Critical Care Medicine. J Am Soc Echocardiogr. 2007;20(7).

18. Sigmund AE, Stevens ER, Blitz JD, Ladapo JA. Use of Preoperative Testing and Physicians' Response to Professional Society Guidance. JAMA Intern Med. 2015;175(8):1352-9.

\section{Submit your next manuscript to BioMed Central and we will help you at every step:}

- We accept pre-submission inquiries

- Our selector tool helps you to find the most relevant journal

- We provide round the clock customer support

- Convenient online submission

- Thorough peer review

- Inclusion in PubMed and all major indexing services

- Maximum visibility for your research

Submit your manuscript at www.biomedcentral.com/submit

C) Biomed Central 